\title{
Resolved Shot Noise in the AM Her System V834 Cen
}

\author{
Stefan Larsson, Bengt Larsson \\ Stockholm Observatory, S-13336 Saltsjöbaden, Sweden
}

\section{Introduction}

Cataclysmic variables of the AM Her type are close binary systems with accretion onto a highly magnetic (a few times $10^{7} \mathrm{G}$ ) white dwarf. In these systems the magnetic field is strong enough to prevent the formation of an accretion disc. The accretion flow is instead funneled directly onto the polar caps where the plasma is shocked and radiates intense optical cyclotron radiation as well as bremsstrahlung at $\mathrm{X}$-ray energies. The cyclotron radiation component, which dominates in the optical, is highly polarized (5-35\%). The cyclotron emission exhibits intensity variations on a large range of time scales. This include quasiperiodic oscillations, flickering and long term changes between brightness states. The observed source intensity is also modulated by the white dwarf rotation which is synchronized with the orbital period (101 min for V834 Cen). The flickering is believed to be due to inhomogeneities in the accretion flow. These could either be density variations in a continous flow or it could be in the form of independent blobs accreting over the polar cap region. As blobs hit the white dwarf surface and radiate their accretion energy they should be seen as shot noise. For a theoretical discussion of the fate of such blobs see Frank et al. (1988). A modeling of the optical variability in AM Herculis, in terms of a shot noise process was made already by Panek (1980). It was found that a consistent model could be constructed by randomly occurring $70^{8}-90^{\mathrm{s}}$ rectangular shots, with on the average a few overlapping shots. The power spectrum had a $\nu^{-2}$ shape above $0.02 \mathrm{~Hz}$ as expected, although it is not clear whether a break in the slope of the power spectrum was actually seen around $0.01 \mathrm{~Hz}$. The shot overlap meant that individual shots could not be resolved directly in the light curve. This limits the precision and uniqueness by which shot parameters can be determined. However, in our observational data of AM Her objects we have found occasions when the character of the flickering has changed to become much more "flarelike", suggesting that individual shots might be seen in these cases. This change is also associated with a change in the shape of the power spectrum, in particular a higher power level and a steepening at the high frequency end. Most importantly, there is a distinct break in the power spectra around $0.03 \mathrm{~Hz}$, 
indicative of a characteristic time scale in the flickering noise. In addition to a power spectrum analysis we have also made a direct modeling of individual flares in the light curve. The distribution and correlations of the fitted pulse parameters were studied and compared with simulated data. The primary aim was to investigate whether the flares are really individual shots or produced by a random superposition of more frequent smaller amplitude shots.

\section{Observations and analysis}

The observations of V834 Cen were made with the $3.6 \mathrm{~m}$ and the Danish $1.5 \mathrm{~m}$ telescopes at ESO at four different occasions from 1984 to 1987. The initial aim of the observations was to study the $\sim 2$ second quasi-periodic oscillations in this object and those results have already been published by Larsson (1992). During 5 nights of observation in January 1987, however, the object also exhibited the distinct flickering which appears to be resolved into individual pulse-shots. One orbital period was observed each night, with a time resolution of $40 \mathrm{~ms}$. An example of one of these light curves can be found in Larsson (1990).

The following basic analysis was made on the time series data for V834 Cen:

- All peaks in the light curves with some height above the noise level were identified and analysed by directly fitting a few different pulse shapes. The distribution of pulse parameters, such as shape, amplitude and width were then analysed and compared to the corresponding results for simulated data.

- Power spectra were calculated from $1500 \mathrm{sec}$ data segments. These were then averaged for all the five nights to produce an overall mean power spectrum.

\section{Results}

\subsection{Fitting individual pulses}

The fitting of individual pulses gave the following primary results:

- The distribution of pulse parameters indicates the presence of a distinct flickering component consisting of high amplitude narrow pulses.

- The distinct shots are best fitted by peaked pulses, such as triangular or $\exp \left\{-\left|\left(t-t_{0}\right) / \tau\right|\right\}$. The asymmetry of the individual pulses were calculated in terms of the rise and decay timescales as $\left(\tau_{+}-\tau_{-}\right) /\left(\tau_{+}+\tau_{-}\right)$. The mean asymmetry was close to zero but had a distribution with an rms deviation of $\sim 0.3$.

- The Full Width Half Maximum (FWHM) distribution for the distinct shots is strongly peaked around $6-7 \mathrm{sec}$. The distribution is shown in Fig. 1 together with an empirical distribution function fitted to the data.

- The amount of overlap for the distinct shots is small.

The result in the last point is suggested both by the observed separation in the light curve and more quantitatively by parameter distribution and correlations (e.g. between amplitude and width). Such correlations were compared with those from simulations with different amounts of overlap. 


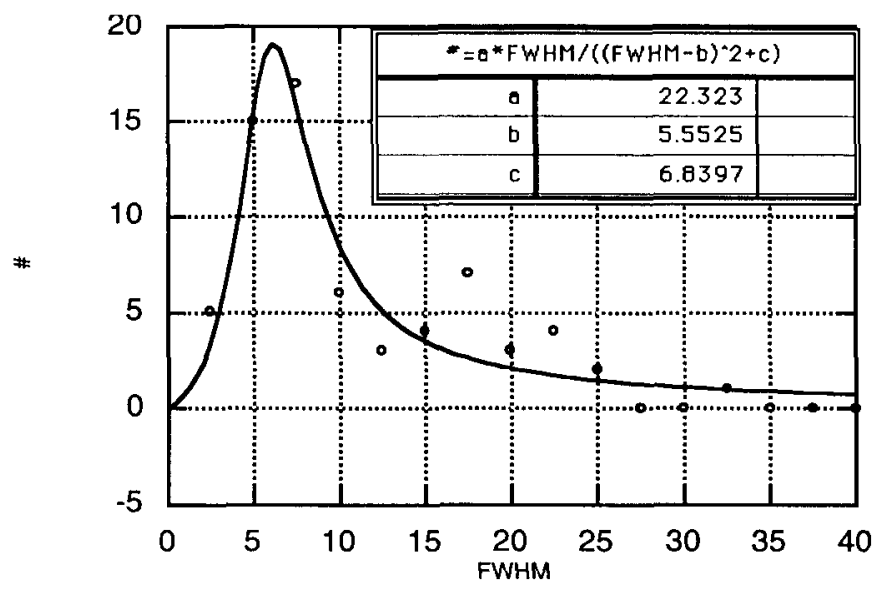

Fig. 1. The Full Width Half Maximum (FWHM) distribution for the distinct shots as determined from the fitting of pulse profiles directly to the time series data. The full drawn curve is a simple parametrization of the distribution which is used to construct a shot noise model for the overall power spectrum.

\subsection{The overall power spectrum}

The total average power spectrum for the 5 nights that exhibited the anomalous flickering is shown in Fig. 2. The most important feature is the break at about $0.03 \mathrm{~Hz}$, which provides strong support for a shot noise model. Above this frequency the spectrum steepens to a power law of slope -2.5 . This is significantly steeper than the "canonical" value of -2 that is produced by e.g. sharp edged shots, such as one-sided exponentials. For rectangular pulses the power spectrum has a $\sin c^{2}$ form and therefore an envelope of $\nu^{-2}$.

\section{Discussion and model}

After the extraction of pulse parameters for what appears to be a distinct shot noise component we now proceed by investigating whether the parameters are consistent with the observed time averaged power spectrum. First however, we want to point out that the pulse profiles implied by the fits in Sect. 3.1 (e.g. double sided exponentials) correspond to very steep power spectra, $\nu^{-4}$ ! We have confirmed this by calculating power spectra for the individual observed 'flares'. These were then averaged in three groups with regard to pulse width. Each of these groups had power law slopes close to -4 as expected. We then calculated the power spectrum produced by double-sided exponentials with a distribution in FWHM taken from the fit in Fig. 1. The distribution in pulse widths flattens the power spectrum slope at high frequencies from -4 to -2.4 which is very close to that of the average spectrum as can be seen in Fig. 2 . The turn over around $\log (\nu)=-1.6$ is also reproduced by this model. The excess 


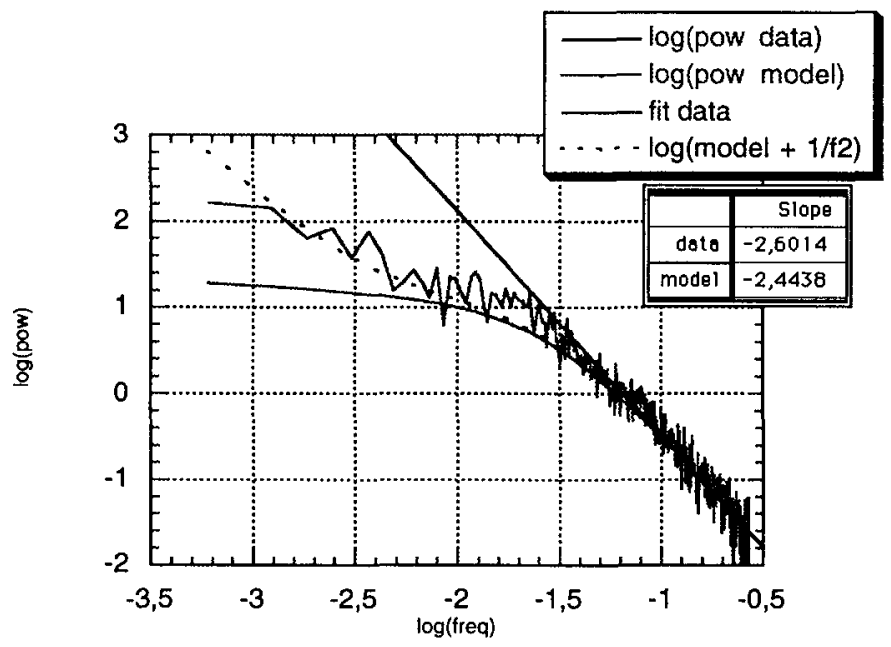

Fig. 2. The average power spectrum for the observations of V834 Cen in January 1987. The straight line is a power law fit to the steep high frequency part of the spectrum. The second solid curve, which is almost flat below $\log (\nu)=-2$, is a model consisting of double-sided exponential shots with a distribution in FWHM taken from the fit in Fig. 1. The dotted line is the same model but after adding a $\nu^{-2}$ component.

over the model at frequencies below $\log (\nu)=-2$ can be explained by adding a $\nu^{-2}$ component with the same strength as in our previous observations of V834 Cen in 1984 and 1985. The only parameter that was adjusted to obtain the fit in Fig. 2 was the strength of the shot noise component.

\section{References}

Frank J, King A.R., Lasota J.-P., 1988, A\&A 193, 133

Larsson S., 1990, in 'Accretion-Powered Compact Binaries', C. Mauche (ed.), Cambridge University Press, Cambridge, p. 279

Larsson S., 1992, A\&A 265, 133

Panek R., 1980, ApJ 241, 1077

A. Bruch: What is the reason for the different time scales of the flickering variations in nights when individual events are not resolved compared to the time scale of the resolved shots?

S. Larsson: This does probably represent a change in the amout, or scale of fragmentation in the accretion flow. 